\title{
Combined Astigmatic Arcuate Keratotomy with Descemet Automated Endothelial Keratoplasty
}

\author{
Jacques Bijon Myrsini Petrelli Basile Salmon Kattayoon Hashemi \\ George D. Kymionis \\ Department of Ophthalmology, Jules-Gonin Eye Hospital, Fondation Asile des Aveugles, \\ University of Lausanne, Lausanne, Switzerland
}

\section{Keywords}

Astigmatic arcuate keratotomy · Descemet automated endothelial keratoplasty · Endothelial decompensation $\cdot$ Postoperative astigmatism

\begin{abstract}
We report the results of simultaneous astigmatic arcuate keratotomy (AK) and Descemet automated endothelial keratoplasty (DSAEK). A 55-year-old patient with a history of high myopia was referred for the management of bullous keratopathy secondary to an anterior chamber phakic intraocular lens ( $\mathrm{plOL}$ ). IOL explantation through a $5.5-\mathrm{mm}$ corneal incision, cataract extraction, and posterior chamber IOL implantation, combined with DSAEK, were performed. Postoperatively, increased astigmatism up to 2.0 diopters (Dpt) was observed, attributed to the large corneal incision, and remained stable, despite suture removal at 3 months. One year postoperatively, the graft showed signs of progressive endothelial dysfunction. A combined procedure of astigmatic AK and DSAEK was thus performed. After 6 months, topographic astigmatism was significantly reduced to $0.5 \mathrm{Dpt}$ and best-corrected visual acuity increased. In conclusion, simultaneous astigmatic AK and DSAEK could be an effective combination for treating patients with well-documented pre-existing astigmatism and endothelial decompensation.
\end{abstract}

\section{Introduction}

Severe endothelial loss and consequent pseudophakic bullous keratopathy represent rare, yet severe, complications following phakic intraocular lens (pIOL) implantation for the correction of high refractive errors. More precisely, the Artisan (Artisan, Ophtec BV) IOL 
phakic iris-claw implant has been associated with significant, long-term corneal endothelial cell loss $[1,2]$. In patients who develop pIOL-related endothelial decompensation, a combined surgical approach involving pIOL removal, phacoemulsification, posterior chamber IOL implantation, and endothelial keratoplasty represents a favorable treatment option [3]. Descemet automated endothelial keratoplasty (DSAEK) has become the mainstay of surgical treatment for addressing corneal endothelial dysfunction [4]. Although it is a minimally invasive surgical technique with minor postoperative refraction changes [4], large corneal incisions, required in cases where DSAEK is combined with rigid pIOL removal, are known to induce astigmatism [5, 6]. To address post-keratoplasty astigmatism, both femtosecond laser and manual astigmatic arcuate keratotomy (AK) have been shown to be effective methods [7-9] with no significant differences in terms of postoperative outcomes [10].

Herein, we describe a combined procedure of astigmatic AK and DSAEK for the treatment of concurrent pre-existing corneal astigmatism and endothelial dysfunction in a patient with previous pIOL explantation. To our knowledge, this combined procedure is the first reported in the literature.

\section{Case Report}

A 55-year-old female patient with a history of high myopia and Artisan pIOL implantation 15 years prior was referred to our clinic for the management of pseudophakic bullous keratopathy in the left eye. Upon presentation, best-corrected visual acuity (BCVA) was 0.1 (decimal scale) in the involved eye, while slit-lamp examination revealed severe bullous keratopathy in the presence of an anteriorly fixated iris-claw Artisan IOL. The right eye status was within normal limits. Dilated fundus examination showed no signs of posterior segment pathology in both eyes. Corneal topography (Pentacam, Oculus Inc., Wetzlar, Germany) showed a central corneal thickness (CCT) of $683 \mu \mathrm{m}$. We decided to perform pIOL extraction through a 5.5-mm superior clear corneal incision, phacoemulsification, and posterior chamber IOL (Acrysof IQ SN60WF + 10D) implantation in the bag followed by a DSAEK procedure (graft thickness of $102 \mu \mathrm{m}$ ) through the same main incision. Corneal sutures were removed at 3 months after surgery. At 6 months postoperatively, BCVA increased to 0.3 ( $+0.5 \mathrm{Dpt} ;-3.0$ Dpt at $80^{\circ}$ ). Slit-lamp examination showed a clear cornea with a functional graft. The topographic and refractive astigmatism were 2.0 Dpt and $-3.0 \mathrm{Dpt}$, respectively, with a CCT of 639 $\mu \mathrm{m}$. It is noteworthy that the past ocular history of the patient prior to presentation showed a maximum BCVA of 0.8 .

Twelve months postoperatively, the patient reported ongoing visual discomfort. The cornea started showing signs of endothelial dysfunction with a continuous increase in CCT (up to $656 \mu \mathrm{m}$ ). In an attempt to address both increased postoperative astigmatism and corneal endothelial dysfunction, we decided to perform a combined surgery of astigmatic AK, followed by DSAEK.

\section{Surgical Technique (online suppl. Video)}

The procedure was performed under retrobulbar anesthesia. The left eye was prepared and draped in a sterile manner. Incisional AK was first created with a diamond micrometer blade. Two arcuate incisions were made at the steep corneal meridian with an angular length of $70^{\circ}$, aiming a depth of approximately $90 \%$ of the corneal thickness (Fig. 1a, b), and a 7-mm corneal optical zone diameter, using a nomogram for naturally occurring astigmatism of 2.6-3.6 Dpt (based on preoperative patient's refractive astigmatism of -3.0 Dpt,

\section{Karger'}


Fig. 1. Postoperative slit-lamp photo (a) and anterior segment OCT (Heidelberg Engineering, Heidelberg, Germany) (b) showing the manual astigmatic AK (dotted arrow) and the endothelial graft (plain arrow). AK, arcuate keratotomy.
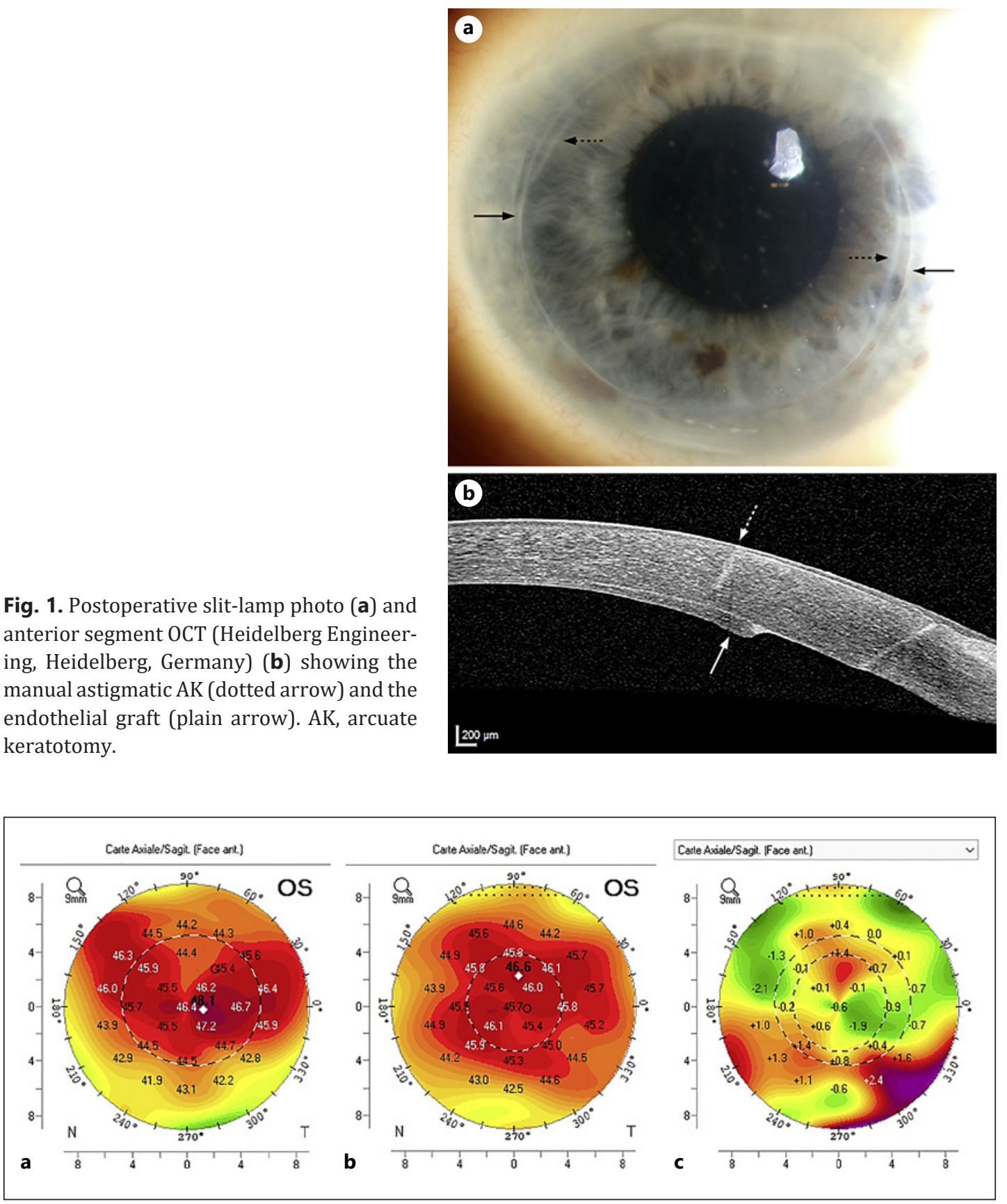

Fig. 2. Preoperative (a) and postoperative (b) corneal topography (Pentacam, Oculus Inc., Wetzlar, Germany) with comparison (c), showing a decrease in postoperative astigmatism.

before the development of corneal edema). The dysfunctional graft was then removed and exchanged with an ultrathin graft of $75 \mu \mathrm{m}$. After suturing the superior 3.2-mm main corneal incision, a bubble of air was injected in the anterior chamber. The postoperative course was uneventful.

At 6-month follow-up, BCVA was $0.4\left(-1.0 \mathrm{Dpt} ;-0.75 \mathrm{Dpt}\right.$ at $\left.130^{\circ}\right)$, while the cornea was clear with no signs of graft dysfunction (Fig. 1a). Topographic and refractive astigmatism were $0.5 \mathrm{Dpt}$ (Fig. 2) and $-0.75 \mathrm{Dpt}$, respectively, while CCT was $568 \mu \mathrm{m}$. 
Bijon et al.: Combined Astigmatic AK with DSAEK

\section{Discussion}

DSAEK offers the advantage of minimum induced astigmatism, as previously described in the literature [4]. In patients with corneal dysfunction, cataract, and significant amounts of astigmatism, simultaneous endothelial keratoplasty, phacoemulsification, and toric IOL implantation remain viable options $[11,12]$. However, in cases where only a DSAEK procedure is required (with no concurrent lens implantation), the management of coexisting astigmatism remains a challenge.

Astigmatic AK, consisting of corneal relaxing incisions at the steep meridian of the midperipheral cornea, is considered an effective surgical procedure for reducing astigmatism. Traditionally, the incisions are made with a diamond micrometer blade. More recently, femtosecond laser has been proposed as an alternative, offering possibly a safer and more predictable AK procedure. Nevertheless, in a recent study that compared the 2 techniques, Al-Qurashi et al. [10] found no significant differences in terms of postoperative BCVA, refractive cylinder and keratometric astigmatism. Overall, regardless of the technique used for incisions, it has been shown that $\mathrm{AK}$ allows a statistically significant improvement in topographic and refractive astigmatism [10], and it is considered a safe procedure in the treatment of postkeratoplasty astigmatism [8]. In our case, manual astigmatic incisions were selected, hence allowing this double surgical intervention to be performed simultaneously in the operating room and to reduce the overall cost of the operation.

Previous studies have described surgical techniques combining astigmatic AK with different anterior segment procedures, such as cataract extraction, with satisfactory postoperative results regarding safety and astigmatism reduction [13]. Similarly, we combined astigmatic AK and DSAEK for the treatment of pre-existing, surgically induced astigmatism and corneal endothelial dysfunction. This simultaneous approach would benefit the patient by hastening visual recovery and reducing the overall cost of the operation as mentioned earlier. In our case, the $5.5-\mathrm{mm}$ corneal incision, required for the removal of the rigid pIOL, led to a significant increase in the postoperative astigmatism of our patient. In order to plan an accurate AK procedure, it is essential to have a complete record of patient's refraction and astigmatism (both topographic and refractive) before the onset of endothelial decompensation and the development of corneal edema as the latter can significantly modify the refractive status. The astigmatism of our patient had been well-evaluated and documented before the development of the corneal edema and remained relatively stable, despite the mild worsening of the latter. Therefore, the calculations made for the AK were based on the patient's astigmatism prior to endothelial decompensation. Nevertheless, if these data were not available, a second, separate AK procedure at a subsequent time would have been required in order to achieve a precise refractive outcome.

As a result of this combined procedure, 6 months postoperatively, the cornea was clear with no signs of dryness. Topographic astigmatism improved from -2.0 Dpt preoperatively (before endothelial decompensation) to $-0.5 \mathrm{Dpt}$ (Fig. 2), while refractive astigmatism decreased from -3.0 Dpt to -0.75 Dpt. BCVA increased up to 0.4, and gradual further improvement would be expected over time [14].

In conclusion, to the best of our knowledge, this is the first report of simultaneous astigmatic AK and DSAEK in the literature. Well-documented pre-existing refractive and astigmatism data, prior to the development of corneal edema, are essential when considering this combined technique. Moreover, our approach may be more suitable for cases of UT-DSAEK and Descemet membrane endothelial keratoplasty that induce relatively minor refractive changes compared to the relatively less predictable refractive outcomes of DSAEK [15].

\section{Karger'}


Bijon et al.: Combined Astigmatic AK with DSAEK

\section{Statement of Ethics}

Ethical approval was not required for this study in accordance with national guidelines. Written informed consent was obtained from the patient for publication and any accompanying images and videos.

\section{Conflict of Interest Statement}

All authors have no conflicts of interest to declare.

\section{Funding Sources}

The authors received no financial support for the research, authorship, and/or publication of this article.

\section{Author Contributions}

Design and methodology: all authors. Data acquisition and interpretation: all authors. Original draft preparation: J.B. and B.S. Comments on previous versions of the manuscript: all authors. Critical revision of manuscript: M.P., K.H., and G.K. Figures and video editing: J.B. and B.S. Supervision: M.P., K.H., and G.K. All authors read and approved the final manuscript for publication.

\section{Availability of Data and Material}

All data analysed during this study are included in this article and its see online suppl. material; for all online suppl. material, see www.karger.com/doi/10.1159/000517741 files. Further enquiries can be directed to the corresponding author.

\section{References}

1 Galvis V, Villamil JF, Acuña MF, Camacho PA, Merayo-Lloves J, Tello A, et al. Long-term endothelial cell loss with the iris-claw intraocular phakic lenses (Artisan®). Graefe's Arch Clin Exp Ophthalmol. 2019;257(12):2775-87.

2 Jonker SMR, Berendschot TTJM, Ronden AE, Saelens IEY, Bauer NJC, Nuijts RMMA. Long-term endothelial cell Loss in patients with artisan myopia and artisan toric phakic intraocular lenses: 5- and 10-year results. Ophthalmology. 2018;125(4):486-94.

3 Nahum Y, Busin M. Quadruple procedure for visual rehabilitation of endothelial decompensation following phakic intraocular lens implantation. Am J Ophthalmol. 2014;158:1330-34.e1.

4 Kymionis GD, Mikropoulos DG, Portaliou DM, Boboridis KG, Voudouragkaki IC, Dragoumis ND, et al. New perspectives on lamellar keratoplasty. Adv Ther. 2014;31(5):494-511.

5 Beltrame G, Salvetat ML, Chizzolini M, Driussi G. Corneal topographic changes induced by different oblique cataract incisions. J Cataract Refract Surg. 2001;27:720-7.

6 Merriam JC, Zheng L, Merriam JE, Zaider M, Lindström B. The effect of incisions for cataract on corneal curvature. Ophthalmology. 2003;110:1807-13.

7 Hoffart L, Touzeau O, Borderie V, Laroche L. Mechanized astigmatic arcuate keratotomy with the Hanna arcitome for astigmatism after keratoplasty. J Cataract Refract Surg. 2007;33:862-8.

8 Poole TRG, Ficker LA. Astigmatic keratotomy for post-keratoplasty astigmatism. J Cataract Refract Surg. 2006; 32:1175-9.

9 Bochmann F, Schipper I. Correction of post-keratoplasty astigmatism with keratotomies in the host cornea. J Cataract Refract Surg. 2006;32:923-8. 
Bijon et al.: Combined Astigmatic AK with DSAEK

10 Al-Qurashi M, Al Sabaani N, Al Malki S. Comparison of manual and femtosecond laser arcuate keratotomy procedures for the correction of post-keratoplasty astigmatism. Saudi J Ophthalmol. 2019;33(1):12-7.

11 Yokogawa H, Sanchez PJ, Mayko ZM, Straiko MD, Terry MA. Astigmatism correction with toric intraocular lenses in descemet membrane endothelial keratoplasty triple procedures. Cornea. 2017;36:269-74.

12 Scorcia V, Lucisano A, Beltz J, Busin M. Combined descemet-stripping automated endothelial keratoplasty and phacoemulsification with toric intraocular lens implantation for treatment of failed penetrating keratoplasty with high regular astigmatism. J Cataract Refract Surg. 2012;38:716-9.

13 Wang L, Misra M, Koch DD. Peripheral corneal relaxing incisions combined with cataract surgery. J Cataract Refract Surg. 2003;29(4):712-22.

14 Li JY, Terry MA, Goshe J, Davis-Boozer D, Shamie N. Three-year visual acuity outcomes after Descemet's stripping automated endothelial keratoplasty. Ophthalmology. 2012;119:1126-9.

15 Yokogawa H, Sanchez PJ, Mayko ZM, Straiko MD, Terry MA. Corneal astigmatism stability in descemet membrane endothelial keratoplasty for fuchs corneal dystrophy. Cornea. 2016 May 9;35(7):932-7. 